\title{
Chaotic quantum ratchets and filters with cold atoms in optical lattices: Analysis using Floquet states
}

\author{
G. Hur, C. E. Creffield, P. H. Jones, and T. S. Monteiro \\ Department of Physics and Astronomy, University College London, Gower Street, London WC1E 6BT, United Kingdom
}

(Received 11 November 2004; published 6 July 2005)

\begin{abstract}
Recently, cesium atoms in optical lattices subjected to cycles of unequally spaced pulses have been found to show interesting behavior: they represent an experimental demonstration of a Hamiltonian ratchet mechanism, and they show strong variability of the dynamical localization lengths as a function of initial momentum. The behavior differs qualitatively from corresponding atomic systems pulsed with equal periods, which are a textbook implementation of a well-studied quantum chaos paradigm, the quantum $\delta$-kicked rotor $(\delta$-QKR). We investigate here the properties of the corresponding eigenstates (Floquet states) in the parameter regime of the recent experiments and compare them with those of the eigenstates of the $\delta$-QKR at similar kicking strengths. We show that by studying the properties of the Floquet states we can shed light on the form of the observed ratchet current, as well as variations in the dynamical localization length.
\end{abstract}

DOI: $10.1103 /$ PhysRevA.72.013403

PACS number(s): 32.80.Pj, 05.45.Mt, 05.60.-k

\section{INTRODUCTION}

Periodically kicked quantum systems, such as the $\delta$-kicked rotor $(\delta$-KR $)$, have long played a central role in studies of quantum chaos and the correspondence between quantum behavior and the underlying classical dynamics $[1,2]$. Advances in the manipulation of cold atoms have permitted the experimental realization of these systems in pulsed optical lattices [3]. Experiments with sodium and cesium atoms have demonstrated the phenomenon of "dynamical localization" (DL) - the quantum suppression of classical chaotic diffusion-and established the suitability of these systems as an arena for the study of effects arising from quantum chaos.

When treating conservative quantum systems it is frequently useful to study the system's energy eigenstates, and for periodically driven systems the appropriate generalization of these states is given by the Floquet states. This approach has provided extensive insight into the properties of the standard quantum KR (QKR), and has shown, for example, that DL arises directly from the exponential localization of the system's Floquet states [2]. Observed momentum oscillations associated with chaos-assisted tunneling, in experiments using periodically driven cold atoms [4] and BoseEinstein condensates [5] have also been analyzed with Floquet theory; it was found that the oscillation period is determined by the splittings of the Floquet phases of a pair of symmetry-related eigenstates. The statistics of the QKR Floquet quasienergy spectrum have been studied extensively (see, e.g., [6]) and compared with the predictions of random matrix theory. Notably, though, the $\delta$-QKR has Poissonian statistics (which are typically associated with integrable dynamics) even for very large values of $K$, where the dynamics is fully chaotic. This has been shown to be a further consequence of DL [6].

However, a series of recent theoretical $[7,8]$ and experimental [9-11] studies of cold atom systems subjected to repeating cycles of unequally spaced kicks revealed dynamics rather different from that found in the corresponding stan- dard QKR systems. Two types of unequally $\delta$-kicked systems were investigated. The first $[8,9]$ consists of a $\delta$-KR with small perturbations made to the kick period. We term it the perturbed-period KR. In the second system [11], the system is periodically subjected to pairs of closely spaced kicks. This is referred to as the double $\delta$-KR or $2 \delta$-KR.

In a theoretical study, the perturbed-period KR was found to yield a ratchet current even in the chaotic regime $[7,8]$. This was unexpected in a chaotic Hamiltonian system, since to date only mixed phase-space ratchet mechanisms had been investigated [12,13]. A simple definition of a ratchet is a spatially periodic device which produces a current without net bias (i.e., the time- and space-averaged forces are zero). Most of the extensive ratchet literature deals with dissipative or Brownian ratchets [14] and comparatively little theoretical work has been undertaken on Hamiltonian ratchets, which are dissipation and noise-free.

In Refs. $[9,10]$ a momentum distribution with a nonzero average (constant in time) was obtained experimentally from an atomic cloud with initial zero average momentum. We are unaware of any other experimental studies of Hamiltonian quantum ratchets: all implementations to date have been of dissipative or Brownian ratchets. Hence the results from [10] and also reproduced here represent the only experimental realization of a Hamiltonian quantum ratchet, whether chaotic or mixed phase space, to our knowledge. In Ref. [8] it was also proposed that the chaotic diffusive properties of the perturbed-period KR could be exploited to filter cold atoms according to their momenta, by controlling the dynamical localization.

For the second system, the $2 \delta$-KR, a theoretical and experimental study [11] revealed that the diffusion is dominated by long-ranged correlations which control escape from well-defined momentum trapping regions. This combination of strong chaotic diffusion and long-ranged correlations is unusual, since strong chaos is generally associated with rapidly decaying correlations.

It is clear that Floquet theory is central to the analysis of chaotic, time-periodic quantum systems. The need to under- 
stand further the chaotic Hamiltonian ratchet as well as the $2 \delta$-KR motivated this study of the Floquet states of these systems. The paper is organized as follows. In the next section we review the well-known $\delta$-KR, then introduce the perturbed $\delta$-KR and the double $\delta$-KR systems. In Sec. III we give a brief review of the Floquet approach. In Sec. IV we compare the results with recently obtained experiments on these systems. Finally in Sec. V we give our conclusions.

\section{INTRODUCTION TO $\delta$-KICKED SYSTEMS}

The Hamiltonian for the usual $\delta$-KR can be written as

$$
H=\frac{p^{2}}{2}+K \sin x \sum_{n} \delta(t-n T)
$$

where $K$ is the kick strength and $T$ is the kick period. It is usual, in both experimental and theoretical studies, to work in a system of rescaled units, where for instance, time is measured in units of $T$ (see, for example, $[10,16]$ for details). Without loss of generality, here we take $T=1$.

Consider the evolution of an ensemble of particles with a Gaussian momentum distribution centered on $p_{0}, N(p)$ $=\exp \left[-\left(p-p_{0}\right)^{2} / \Delta p^{2}\right]$. The classical dynamics depends only on the parameter $K$, and for values larger than $K \simeq 1$, the chaotic diffusion is not bounded by classical barriers. In this regime the ensemble will diffuse in momentum space, its average energy growing linearly with time as $\left\langle p^{2}\right\rangle=D t$, where, to lowest order, the diffusion rate is given by $D_{0}$ $=K^{2} / 2$. The distribution will thus remain Gaussian, although its width will increase with time as $\Delta p(t)=\sqrt{D t}$.

In contrast, the quantum system only follows this behavior up to a time scale $t^{*} \simeq D / \hbar^{2}$ [15], after which diffusion in momentum space is suppressed-dynamical localization. Such a system will asymptotically evolve toward a characteristic exponential momentum distribution, $N(p) \sim \exp [-\mid p$ $\left.-p_{0} \mid / \Delta p_{Q}\right]$, with constant width $\Delta p_{Q} \sim \sqrt{D t^{*}} \sim D / \hbar$, which thus acts as an experimental fingerprint for DL [3]. As DL is a wave-coherent effect, the quantum system must preserve coherence over at least the time scale $t^{*}$ for this effect to be observable. Indeed, it has been verified experimentally [16] that the DL profile does not survive the presence of noise or dissipation, and that with decoherence a more Gaussian profile for $N(p)$ is produced.

In both classical and quantum cases the behavior of the standard $\delta$-KR is essentially independent of $p_{0}$ since, even for modest values of $K$, the effects of small fluctuations in the structure of phase-space are on negligible scales relative to $\Delta p_{Q}$. Even if there are small stable islands, they are of size $\Delta p \sim 1$ so have little effect on the general form of $N(p)$, since typically $\Delta p_{Q} \gg 1$.

The classical dynamics of the $\delta$-KR is obtained by iterating the well-known standard map. For the perturbed-period and double $\delta$-kicked systems, on the other hand, the dynamics is given by a two-kick map:

$$
\begin{gathered}
x_{j}=x_{j-1}+p_{j} T_{1}, \\
p_{j+1}=p_{j}-V^{\prime}\left(x_{j}\right),
\end{gathered}
$$

$$
\begin{gathered}
x_{j+1}=x_{j}+p_{j+1} T_{2}, \\
p_{j+2}=p_{j+1}-V^{\prime}\left(x_{j+1}\right) .
\end{gathered}
$$

For the perturbed-period KR, the lengths of the two kicking periods are $T_{1}=1+\epsilon$ and $T_{2}=1-\epsilon$, where $\epsilon \ll 1$. The perturbation thus consists of slightly altering the kicking period about its mean. For the double $\delta$-KR we take $T_{1}=2-\epsilon, T_{2}$ $=\epsilon$, although we shall also show the effect of interchanging the two kick periods. It should be noted that these systems are time periodic, with period $T_{\text {tot }}=T_{1}+T_{2}$, and are thus quite distinct from the recent interesting study of two independent kicking sequences, which can be nonperiodic and hence nonlocalizing [17].

As in the standard map, we consider a sinusoidal potential. However, to obtain a ratchet current in the case of the perturbed-period KR, we need to break the spatiotemporal symmetries, and so we add an additional "rocking" linear potential of strength $A$. In this case the form of the potential is

$$
V(x)=\left[K \cos x+A x(-1)^{j}\right],
$$

where $j$ is the kick number. In experimental implementations of this system, the rocking linear term was obtained by means of an accelerated lattice [9]. Clearly, setting $T_{1}=T_{2}$ $=1$ and $A=0$, we recover the standard map.

We first consider in general terms how the introduction of the second time scale $\epsilon$ modifies the classical behavior of the standard map. If we neglect all correlations, the standard map has a constant momentum diffusion rate $D_{0} \simeq K^{2} / 2$-this is what one would expect if the momenta at consecutive kicks are uncorrelated and so evolve as a random walk. However, unless $K$ is exceedingly large, the time evolution of the standard map will contain some short-range (two- and three-kick) correlations. Including these corrections yields a modified diffusion constant $D=\left(K^{2} / 2\right)\{1$ $\left.-2 J_{2}(K)-\left[J_{1}(K)\right]^{2} \cdots\right\}$. Of particular interest is the $J_{2}(K) K^{2}$ term, representing correlations $\left\langle V^{\prime}\left(x_{j}\right) V^{\prime}\left(x_{j+2}\right)\right\rangle$ between nearest-but-one kicks (the two-kick correlation).

For the modified systems it is also possible to obtain the important correlations analytically $[8,11]$. For instance, for the perturbed-period system the corrected diffusion is given by

$$
D \simeq \frac{K^{2}}{2}\left\{1-2 J_{2}(K) \cos \left(2 p_{0} \epsilon-A\right)-\left[J_{1}(K)\right]^{2} \cdots\right\} .
$$

We see that the modified two-kick correlation now oscillates as a function of the initial momentum $p_{0}$. This effect is clearly most significant for values of $K$ such that $2 J_{2}(K)$ $\sim 1$.

The key point is that perturbing the kick spacings $T$ by a small amount can result in large scale (relative to $\Delta p_{Q}$ $\sim D / \hbar)$ variations in the classical momentum diffusion, and that these are present even in fully chaotic regimes (we take this to mean the absence of visible stable structures on the Poincaré surface of section). For the analysis of experiments, one must now consider a local diffusion rate $D\left(p_{0}\right)$, which 
depends on the initial relative momentum between the atoms and the optical lattice.

In [9], the perturbed-period system was implemented experimentally with a cloud of cesium atoms for the case $A$ $=0$. It was verified that the energy absorbed by the cloud after dynamical localization $\left\langle\left(p-p_{0}\right)^{2}\right\rangle \propto \cos \left(2 p_{0} \epsilon\right)$ as expected. However, $A=0$ corresponds to a symmetric potential. The case $A=\pi / 2$ in the perturbed-period system is particularly interesting since then the momentum diffusion is asymmetric about $p_{0}=0$. This implies that atoms with positive momenta will absorb kinetic energy at different rates from those with momenta of the same magnitude but moving in the opposite direction. This asymmetric momentum diffusion represents a type of fully chaotic momentum ratchet: in other words, roughly equal numbers of particles will diffuse to the left or to the right, but those diffusing to the right, on average, move faster.

In a further experimental study of the perturbed-period KR [10], the rocking potential was implemented by means of an accelerated lattice. It was found that an atomic cloud prepared initially with a Gaussian momentum distribution centered on $p_{0}=0$ evolved into a distribution with nonzero, but constant, $\langle p\rangle$ which persisted even beyond the break time $t^{*}$, as expected from the theory [7]. This type of chaotic directed motion was first identified in a slightly different system: a kicked asymmetric double-well potential [7]. However, the latter potential gives rise to a rather more complicated diffusive behavior, and also has proved much harder to implement experimentally. For these reasons here we do not consider the case of the asymmetric double-well ratchet, but note that our Floquet analysis of the perturbed-period KR can be carried over to the system investigated in [7].

The second system we consider explicitly in this work, the double $\delta$-KR, has diffusive behavior which is qualitatively different to both the standard map and perturbedperiod KRs. While for these other kicked systems we can analyze the diffusion as an uncorrelated term, $K^{2} / 2$, corrected by short-ranged correlations (typically only two- or three-kick correlations for $K \simeq 3$ ), for the double $\delta$-KR, we find that the diffusion at long times is dominated by families [11] of long-ranged "global" correlations ("global" in the sense that they correlate all kicks up to the time under consideration). At short times, the diffusion is dominated by a one-kick correlation not present in other kick systems. At longer times, the global diffusion terms, though weak, accumulate and eventually become dominant.

The method of correlations provides a generic and accurate way of interpreting experimental data for this system [11]. There is also a simple physical picture. For particles subjected to kicks of form $K \sin x$, consecutive kicks will be out of phase and will hence cancel if $p_{0} \epsilon \simeq(2 n+1) \pi$ where $n=0,1,2 \ldots$ In other words, an impulse $V^{\prime}\left(x_{j}\right)=K \sin x_{j}$ will be immediately followed by another which cancels it, since $V^{\prime}\left(x_{j+1}\right)=K \sin x_{j+1} \simeq K \sin \left(\pi+x_{j}\right)$. This cancellation means that particles become trapped at these momenta. In contrast, particles for which $p_{0} \epsilon \simeq 2 n \pi$ will experience enhanced diffusion.

It was shown in [11] that the types of global families of correlations described there control the escape from, and through, these "trapping regions." An unexpected feature of the classical calculations (and also seen in experiment) was the observation that particles initially prepared in the trapping regions will eventually gain more energy than those initially prepared in regions of enhanced diffusion, after a time scale $t \gg 1 /(K \epsilon)^{2}[11]$.

\section{QUANTUM DYNAMICS AND FLOQUET STATES}

If a Hamiltonian has a $T$-periodic time dependence, $H(t$ $+n T)=H(t)$, then the Floquet theorem implies that solutions to the time-dependent Schrödinger equation can be written in the form

$$
\psi(t)=\exp (-i \epsilon t / \hbar) \phi(t)
$$

where $\phi(t)$ is a $T$-periodic function called a Floquet state, and $\epsilon$ is a quantity with dimensions of energy, termed a quasienergy. This type of relation is familiar in the context of solid-state physics, where a Hamiltonian's invariance under discrete shifts of spatial position (typically arising from a lattice structure) allow solutions to be written analogously in terms of Bloch states and quasimomenta. The Floquet states provide a complete basis, and thus the time-evolution of a general quantum state under periodic driving can be expressed as

$$
\Psi(t)=\sum_{n} c_{n} \exp \left(-i \epsilon_{n} t / \hbar\right) \phi_{n}(t),
$$

where $\left\{c_{n}\right\}$ are time-independent expansion coefficients. It is clear from this expression that the Floquet states and quasienergies play a similar role for periodically driven systems to that of energy eigenstates and eigenvalues in the time-independent case.

The time-evolution operator $U\left(t_{2}, t_{1}\right)$ may be used to evolve a quantum state from time $t=t_{1}$ to time $t=t_{2}$. For a time-periodic system, the single-period propagator $U(T, 0)$ allows a quantum system to be evolved "stroboscopically" at intervals separated by the period $T$ with great efficiency, by defining the quantum map $\Psi(n T)=[U(T, 0)]^{n} \Psi(0)$. In terms of Floquet states, it is straightforward to show that the timeevolution operator is given by

$$
\begin{gathered}
U\left(t_{2}, t_{1}\right)=\mathcal{T} \exp \left(-\frac{i}{\hbar} \int_{t_{1}}^{t 2} H\left(t^{\prime}\right) d t^{\prime}\right) \\
=\sum_{n} e^{-i \epsilon_{n} t / \hbar}\left|\phi_{n}\left(t_{2}\right)\right\rangle\left\langle\phi_{n}\left(t_{1}\right)\right|,
\end{gathered}
$$

where $\mathcal{T}$ is the time-ordering operator, and thus it can be seen that the quasienergies and Floquet states can be conveniently obtained by simply diagonalizing the one-period propagator. The eigenvectors of this operator are the Floquet states, while its eigenvalues are related to the quasienergies via $\lambda_{n}$ $=\exp \left(-i \epsilon_{n} T / \hbar\right)$.

Obtaining an explicit form for this propagator is normally a complicated procedure, as in general the driving field does not commute with the static Hamiltonian. For the case of $\delta$ kicking, however, the problem is simplified considerably 
which allows an analytic form for the propagator to be written. For the QKR [Eq. (2.1)] the propagator is given by

$$
U(T, 0)=\exp \left(-i T \hat{p}^{2} / 2 \hbar\right) \exp [-i(K / \hbar) \sin \hat{x}] .
$$

Since the kicking potential is invariant under spatial translations of $2 \pi$, Bloch's theorem permits the eigenvalues of $\hat{p}$ to be decomposed into the form $p_{m}=(m+q) \hbar$, where $m$ is an integer and $q$ is a fraction lying in the interval $[-0.5,0.5)$, termed the "quasimomentum." A true kicked rotor system, as distinct from a kicked particle, would be obtained by setting the quasimomentum $q=0$. Using this basis, the matrix elements $\left\langle m+q|U(T, 0)| n+q^{\prime}\right\rangle$ of the propagator can easily be shown to be

$$
U_{m, n}(T, 0)=\exp \left[-i T(m+q)^{2} / 2 \hbar\right] J_{m-n}(K / \hbar) \delta\left(q-q^{\prime}\right),
$$

where $J_{n}$ is the $n$th Bessel function of the first kind. For practical purposes it is useful to note that $\left|J_{m-n}(x)\right|$ decreases extremely rapidly with increasing $|m-n|$, thus giving $U$ an effectively banded structure.

The one-kick propagators $U\left(T_{1}, 0\right)$ and $U\left(T_{2}, 0\right)$, where $T_{1}$ and $T_{2}$ are the two kick periods, take a slightly different form from Eq. (3.6) if we include a ratchet potential (i.e., $A \neq 0$ ). Nevertheless in all cases the two-kick (full-period) propagator can be expressed as the product $U\left(T_{\text {tot }}, 0\right)=U\left(T_{1}\right.$ $\left.+T_{2}, T_{1}\right) U\left(T_{1}, 0\right)$. Note that for $A \neq 0$, however, although the single-kick propagators do not conserve quasimomentum, their product does. As a consequence, the evolution of an ensemble of noninteracting particles, which can be modeled by the evolution of a superposition of states with different quasimomenta, represents a computationally efficient procedure: since $U\left(T_{\text {tot }}, 0\right)$ is block diagonal in $q$, we can consider each quasimomentum component separately and diagonalize the smaller submatrices.

The matrix representation for the full-period propagator, in its most general form, has elements

$$
\begin{aligned}
U_{l, n}(T, 0)= & i^{n-l} e^{-i T_{1}(l+q)^{2} \hbar} \sum_{j} e^{-i T_{2}(j+q a)^{2} \hbar} \\
& \times J_{l-j+k a}\left(\frac{K}{\hbar}\right) J_{j-n-k a}\left(\frac{K}{\hbar}\right),
\end{aligned}
$$

where $k a=\operatorname{int}(A)$ and $q a=A-k a$. To obtain the form appropriate for the $2 \delta$-KR, it is simply necessary to set $A=0, T_{1}$ $=2-\epsilon$, and $T_{2}=\epsilon$.

Having obtained the Floquet states by diagonalizing the full-period propagator, it is useful to analyze their structure, particularly their spread in momentum space. We do this by evaluating a localization length, $L$ of each Floquet state at $T=0$. We note that in this case we cannot assume that the Floquet states have the usual exponential momentum distribution $N(p) \sim \exp \left(-\left|p-p_{0}\right| / L\right)$, of the usual $\delta$-KR. Hence we take $L$ to be simply the root mean square deviation from the mean, $L=\sqrt{\bar{p}_{n}^{2}}{ }_{n}-\bar{p}_{n}^{2}$. In this expression $\bar{p}_{n}$ is the mean momentum of the $n$th Floquet state at $t=0, \bar{p}_{n}$ $=\left\langle\phi_{n}(0)|\hat{p}| \phi_{n}(0)\right\rangle$, and $\bar{p}_{n}{ }_{n}=\left\langle\phi_{n}(0)\left|\hat{p}^{2}\right| \phi_{n}(0)\right\rangle$. If the Floquet states do not have a strong time dependence, such as for the standard or even the perturbed-period KR, this is adequate to

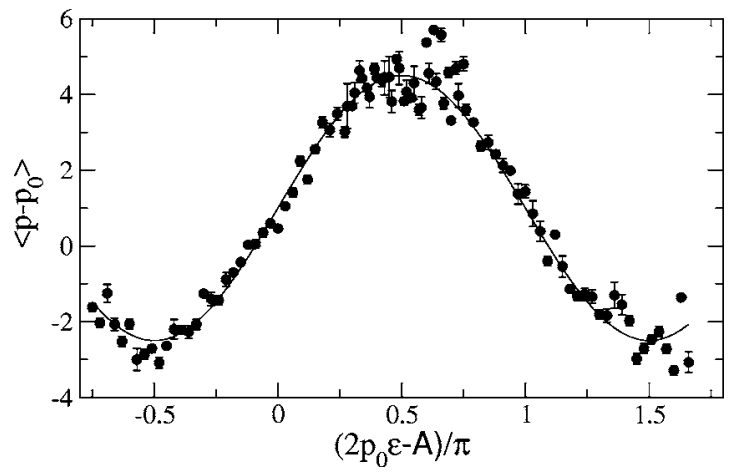

FIG. 1. Experimental values of the ratchet current $I=\left\langle p-p_{0}\right\rangle$, for the perturbed-period $\mathrm{KR}$, obtained with cold cesium atoms in a pulsed optical lattice for $K=2.6, \epsilon=1 / 16$. The solid line is a best fit to the data, showing that the current oscillates sinusoidally as $I$ $\propto \sin \left(2 p_{0} \epsilon-A\right)$.

quantify the degree of spreading in momentum space. We shall see, however, that for the case of the double $\delta$-KR it is not sufficient to measure the localization at just a single time, due to the extremely strong time dependence of the Floquet states.

\section{RESULTS}

\section{A. Perturbed-period KR: Experimental realization of a chaotic ratchet}

Figure 1 shows a plot of the experimental ratchet current obtained in [10] using distributions of cold cesium atoms with $p_{0}=0$, in an optical lattice pulsed with unequal periods. The momentum distribution is essentially unchanged after about 60 kicks; the plotted values correspond to about $T$ $=200$ kicks, hence well after dynamical localization. Full details are given in $[9,10]$, but by employing an accelerated lattice, the experiment was able to simulate an effective rocking potential with a given value of $A$. The current (the first moment of each localized momentum distribution $I=\langle p$ $\left.\left.-p_{0}\right\rangle\right)$ was then calculated, and is plotted as a function of $A$ in Fig. 1.

Figure 1 shows that the ratchet current oscillates sinusoidally, i.e., $I \propto \sin \left(2 p_{0} \epsilon-A\right)$. This can be qualitatively understood from the form of the classical two-kick momentumdiffusion correction [Eq. (2.3)] $C_{2}=-K^{2} J_{2}(K) \cos \left(2 p_{0} \epsilon-A\right)$. If we set $p_{0}=0$, as in the experiment, then for a very small momentum displacement $\delta p$, the differential absorption of energy for particles moving to the left $\delta p<0$ or right $\delta p$ $>0$ is proportional to the gradient $\partial C_{2} / \partial p_{0} \propto K^{2} \sin \left(2 p_{0} \epsilon\right.$ $-A)$. An analytical form for the classical current was derived previously [8]. An asymptotic (small- $\epsilon$ ) approximation for the ratchet current is given by $I \simeq\left[J_{2}(K) / \epsilon\right] \sin \left(2 p_{0} \epsilon-A\right)$, implying the maximal classical current is $I_{\max } \sim J_{2}(K) / \epsilon$.

The results in Fig. 1 correspond to $K=2.6, \epsilon=1 / 16$, and $\hbar=1$ (note that in rescaled units $\hbar=8 \omega_{R} T$, where $\omega_{R}$ is the Rabi frequency). This effective value of Planck's constant may be varied: in the experiments, the range $\hbar \simeq 1 / 4 \rightarrow 1$ was investigated. For these values of $K$ and $\epsilon$, we estimate $I_{\max } \sim J_{2}(K) / \epsilon \simeq 8$, about twice the observed value. This is 
not unreasonable as $\epsilon=1 / 16$ is not so small and we neglect higher-order corrections to the classical current. The experiments in [10] indeed indicate that halving $\epsilon$ almost doubles the maximal current. Note that changing the sign of $\epsilon$ implies a current reversal.

At this stage it may be unclear to the reader what the significance of altering the sign of $\epsilon$ in the experiment might be, since after all, the rocking potential involves alternating impulses $-V^{\prime}(x)=K \sin x \pm A$. In fact the distinction (as may be ascertained when calculating correlations) is between the case where an impulse $-V^{\prime}(x)=K \sin x+A$ follows free evolution for the longer time interval $T_{1}=1+\epsilon$ [and the impulse $-V^{\prime}(x)=K \sin x-A$ follows free evolution for the shorter time interval $\left.T_{2}=1-\epsilon\right]$ and the separate experimental case where an impulse $-V^{\prime}(x)=K \sin x-A$ follows evolution for a time interval $T_{1}=1+\epsilon$ and so forth (which corresponds to an current of identical magnitude but of opposite sign).

Of course, it is clear that a nonzero and persistent constant ratchet current is also obtained classically. It was found in [7] that asymmetric diffusion accumulates only on a time scale $t_{r} \sim 1 /(K \epsilon)^{2}$ (termed the "ratchet time" in [7]). Note that $I_{\max } \sim J_{2}(K) / \epsilon$ implies that $I_{\max } \rightarrow \infty$ as $\epsilon \rightarrow 0$, but in that case $t_{r} \sim \infty$ and so the final value of the current is never reached. For the (unbounded) chaotic system studied here, the acquired momentum asymmetry is never lost. For a bounded ("compact phase-space") system, such asymmetries would vanish on a long time scale, since the distribution of a fully chaotic system would eventually become uniform. For this reason, until recently, it was argued that a fully chaotic system could not generate directed motion. So, although as shown in [7], the fully chaotic classical system can keep a constant current for long times, practical implementation is ultimately less interesting since the average kinetic energy of the ensemble grows linearly with time and without limit. Hence, this type of chaotic ratchet is of most interest as a quantum rather than a classical ratchet since in the quantum case DL halts the diffusion and "freezes in" the asymmetry, without the need for classical barriers like tori.

In Fig. 2 we reproduce two experimental momentum distributions for $K \simeq 3$ obtained with cesium atoms in Ref. [10], for $A= \pm \pi / 2$. We clearly see that the origin of the nonzero current is the asymmetric momentum distribution. As expected, Fig. 2 shows that changing the sign of $A$ reverses the asymmetry and produces a current reversal, in an analogous fashion to the way that altering the sign of $\epsilon$ also reverses the current.

Note that the experimental range of $K \simeq 2.6-3.4$ does correspond to a classical surface of section with some islands. However, we note that classical quantities such as the average energy are very accurately given by diffusion rates (with two- and three-kick corrections). The essential mechanism is asymmetric chaotic diffusion: similar behavior was found at larger $K$ in [7] in regimes where there are no visible classical islands (but for which experiments are not available); hence, in the analysis of this type of ratchet, the presence (or otherwise) of small stable islands is immaterial. What is important, though, is that since the asymmetric diffusion term is $2 J_{2}(K) \cos \left(2 p_{0} \epsilon-A\right)$, we need $J_{2}(K) \neq 0$. Thus the much studied (for the standard map) parameter value $K=5$ does not

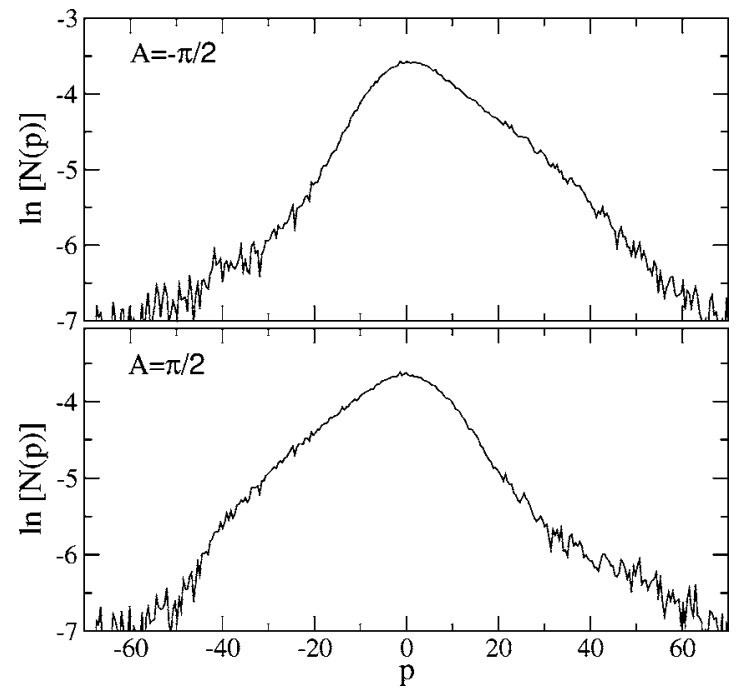

FIG. 2. Experimental momentum distributions $N(p)$ for the perturbed-period $\mathrm{KR}$ obtained with cold cesium atoms in a pulsed optical lattice for $K \simeq 3, \epsilon=1 / 16$. The distributions have localized, and hence remain essentially constant with time. The results show clearly that the origin of the net nonzero value of $\langle p\rangle$ obtained at long times is in the asymmetry of the DL profiles. As expected, the asymmetry is reversed by changing the sign of $A$, the amplitude of the rocking potential.

produce asymmetry, since $J_{2}(5) \simeq 0$. Values of $K \simeq 2.5-3.5$, $\hbar=1 / 4-1$, on the other hand, turned out to be experimentally convenient and produced the strongest asymmetries.

\section{B. Perturbed-period KR: Floquet states}

We now examine the form of the underlying Floquet states. In Fig. 3 we compare the localization lengths for the standard QKR, with those of the perturbed-period KR for $K=3.4, \epsilon=0.01$. The difference is quite striking; while the standard QKR eigenstates are quite uniform across all regions of phase space, the perturbed-period localization lengths oscillate sinusoidally with $\bar{p}$, with a period of $\pi / \epsilon$. Introducing the additional rocking potential with the accelerated lattice $(A=-\pi / 2)$ clearly leads to a $\pi / 2$ shift in the oscillations. Inspecting Fig. 3(c) for $\bar{p} \simeq 0$, we see that for positive momenta the localization lengths are increasing, while for negative momenta, the localization lengths decrease. Note the nearly regular row of states for the standard QKR case with $L \simeq 1$. These correspond to states localized on a series of stable islands separated by $2 \pi$, due to the momentum periodicity of phase-space in that case.

We have chosen a parameter range for which $L \ll \pi / \epsilon$ : that is, the localization length of each state is much smaller than the oscillation in $\bar{p}$. Hence individual Floquet states really do sample "local" diffusion rates. We found that if we move toward a regime where $L \sim \pi / \epsilon$, the conclusions remain valid, but the amplitude of the oscillations is considerably damped. Similarly, if the sign of $J_{2}(K)$ changes, so does the sign of the sinusoidal oscillation.

We now consider the shape of the Floquet states in detail. In Fig. 4 we show the momentum distributions $N(p)$ 


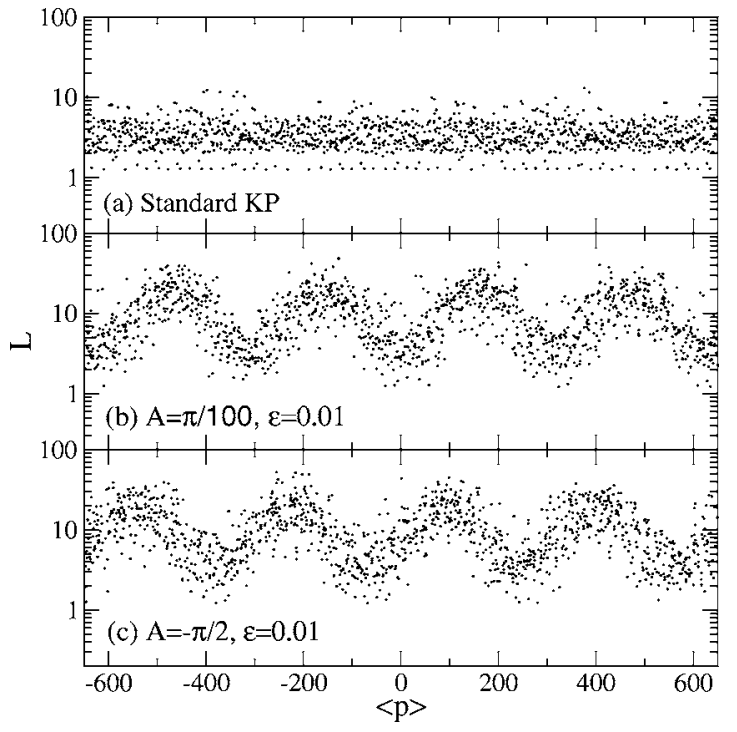

FIG. 3. The graph shows the localization lengths $L$ of Floquet states as a function of average momentum $\bar{p}$ (i.e., $\langle p\rangle$ ) for $K=3.4$, $\hbar=1$. Results are shown (a) for the standard QKR case, i.e., $A$ $=\pi / 100$ (a small but nonzero $A$ was used to break spatial symmetry), $\epsilon=0$, (b) for $\epsilon=0.01, A=\pi / 100$, and (c) for $\epsilon=0.01, A=$ $-\pi / 2$. The graph shows that for the standard kicked rotor the $L$ are distributed within a narrow range in comparison with other two below. For the rocking case, $L$ oscillates with $\bar{p}$ as expected from the two-kick correction $2 J_{2}(K) \cos \left(2 p_{0} \epsilon-A\right)$; the oscillations of the two lower graphs are shifted relative to each other by a phase $\pi / 2$. The density of eigenstates corresponding to average momentum range is roughly the same in all three cases.

$=\left|\phi_{n}(p)\right|^{2}$ for Floquet states of the standard QKR. The distributions (with $N(p)$ on a logarithmic scale) all show the wellknown triangular form [2] - the hall-mark of dynamical localization. It may be clearly seen that the localization lengths vary little from state to state.

In Fig. 5, by contrast, the localization lengths of the Floquet states of the perturbed-period $\delta$-KR display a strong dependence on the mean momentum of the states. In addition, the figure shows that states localized close to $p=0$ are markedly asymmetric. The states are considerably extended toward positive momentum, but are strongly localized to-

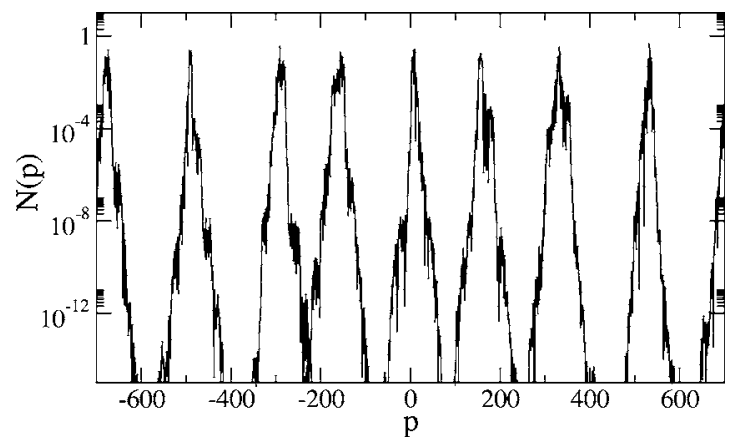

FIG. 4. Floquet states for the standard QKR, for $K=3.4, \hbar=1$. As expected, all the states are exponentially localized, giving the characteristic triangular shape of $N(p)$ when plotted on a logarithmic scale. They all have approximately similar localization lengths.

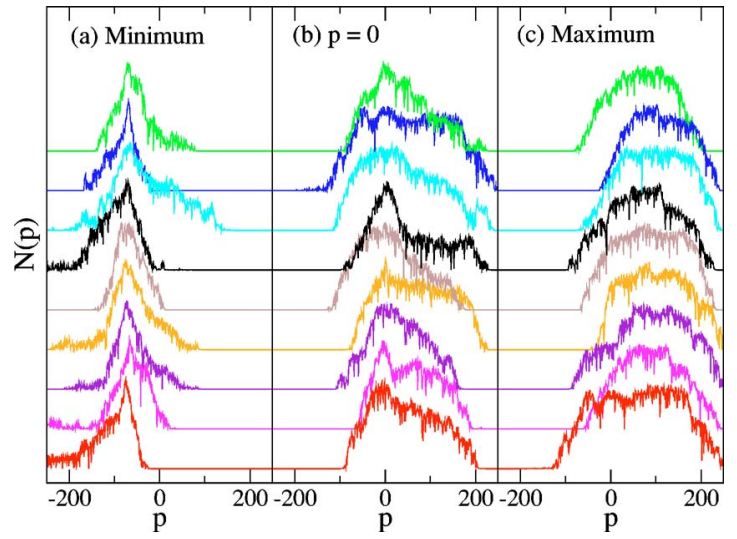

FIG. 5. (Color online) Typical form of Floquet states for the perturbed-period KR, $K=3.4, \epsilon=0.01, A=-\pi / 2$ and $\hbar=1$. Here we plot $N(p)=\left|\phi_{n}(p)\right|^{2}$ on a logarithmic scale as a function of $p$. (a) States with $p \simeq-78$. This corresponds to a minimum of the twokick correction $(2.3)-\cos \left(2 p_{0} \epsilon+\pi / 2\right)$. The states are narrow, but in general roughly symmetric. (b) States with $p \simeq 0$. The typical state here is asymmetric. (c) States with $p \simeq 78$. This corresponds to a maximum of the two-kick correction. States here are generally symmetrical, but broad and flat-topped.

wards negative $p$. This behavior neatly accounts for the form of the experimental momentum distribution shown in Fig. 2, which for $A=-\pi / 2$ were also more extended toward positive $p$. The states localized near $p \simeq-\pi / 4 \epsilon$ and $\pi / 4 \epsilon$ correspond to, respectively, minima and maxima of the classical diffusion. They are roughly symmetrical (typically) but vary by up to a factor of $\sim 40$ in $L$. In Ref. [8] it was proposed that the observed variation in the energy absorption rates between atoms prepared with an initial drift momentum $p_{0}=-\pi / 4 \epsilon$ (which absorb very little energy) and those with $p_{0}=\pi / 4 \epsilon$ might be exploited to filter traffic of atoms through an optical lattice. The form of the underlying Floquet states explains this differential rate of energy absorption.

Subsequently, it was found experimentally that the double $\delta$-KR in fact shows much more pronounced differential absorption rates, without requiring the application of a rocking field $A$. We next report a study of this system.

\section{Double $\delta$-KR: Experimental realization}

A study of the experimental and classical behavior of the double $\delta$-KR was carried out in [11]. The classical dynamics is very different to that of the perturbed-period KR. At very short times, the chaotic diffusion comprises an uncorrelated diffusion term $K^{2} / 2$ and one dominant one-kick correction. It was found in [11] that one can approximate the growth in the mean energy with time $t$, by the simple expression $\left\langle p^{2}\right\rangle$ $\simeq K^{2} t\left[1+\cos p_{0} \epsilon\right]$. In Fig. 6(a) we show experimental results for cesium atoms which localized in this regime. The experiment measured the energy of a series of clouds of $\sim 10^{6}$ atoms moving through the pulsed optical lattice with varying average drift momenta $p_{0}$. For Fig. 6(a), the simple expression given above gives an excellent fit to the experiment, if we take $t \sim t^{*}$, where $t^{*}$ is the break time. This regime corresponds to $t^{*} \ll 1 /(K \epsilon)^{2}$. 


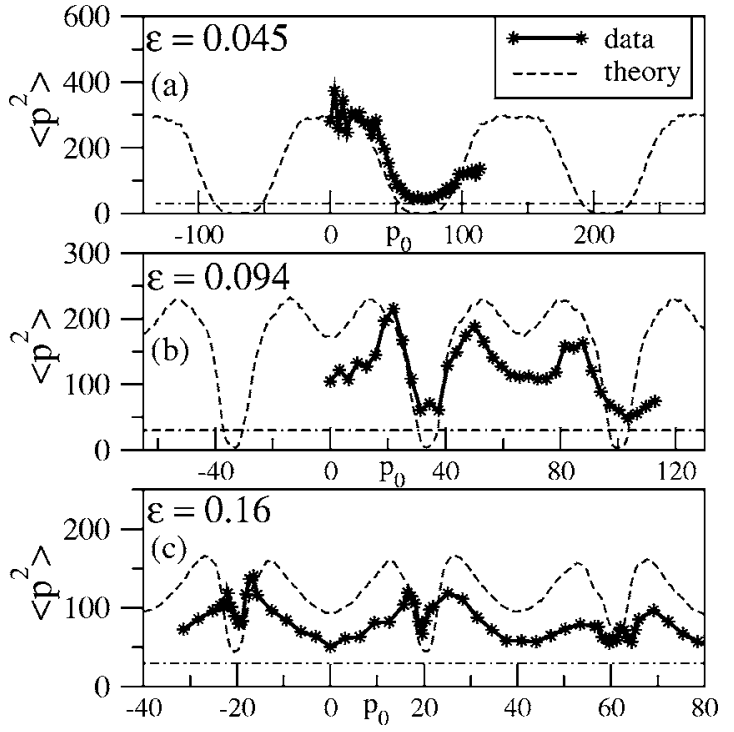

FIG. 6. Experimental results for double $\delta$-KR realization with cesium atoms (see [11] for details). Each data point (star) shows the energy absorbed (after 100 kicks, $K=3.3, \hbar=1$ ) by a cloud of atoms with average momentum $p=p_{0}$ (relative to the optical lattice) at initial time $t=0$. With increasing $\epsilon$, we see the minima (maxima) in the energy flip into maxima (minima) as a long-ranged family of classical correlations gradually overtakes the one-kick classical correlation. The dashed lines represent a classical simulation using 100000 particles, all with momenta $p_{0}$ at $t=0$, and $K$ within the range $3.3 \pm 10 \%$. (a) $t^{*} \ll t_{1} \simeq 1 /(K \epsilon)^{2}$. Regime where a one-kick correlation is the dominant correction to the classical diffusion. Here, atoms prepared near the trapping regions $\left(p_{0} \epsilon \sim(2 n+1) \pi\right)$ remain trapped. Results follow closely the formula $\left\langle p^{2}\right\rangle \simeq K^{2} t(1$ $+\cos p_{0} \epsilon$ ). (b) $t^{*} \sim 1 /(K \epsilon)^{2}$. Regime showing the inverted peaks of the Poisson correlation terms analyzed in [11], which determine the momentum trapping very close to the resonant condition $\left(p_{0} \epsilon\right.$ $=(2 n+1) \pi)$. (c) $t^{*}>1 /(K \epsilon)^{2}$. Regime dominated by correlation family $C_{G 1}$, but sharp inverted peaks due to the Poisson correlations are still visible.

However, a more detailed study of the classical correlations showed that for later times, a new type of correction appeared. Families of long-ranged, or "global," correlations which coupled all kicks appeared. These corrections are individually very weak, but accumulate to eventually dominate the diffusive process. One family (termed the "Poisson family" in [11]) was shown to lead to well-localized, inverted peaks in the energy absorption at values of $p_{0} \simeq(2 n+1) \pi / \epsilon$, where $n=0,1,2, \ldots$. These values of $p_{0}$ correspond to trapping regions in phase space (at low values of $K$, structures corresponding to islands and broken phase-space barriers are evident). However there is no need to investigate detailed transport through this complex mixed phase-space structure, as the correlations give us a generic and quantitative handle on the energy diffusion with time. In this intermediate regime, dominated by the Poisson correlations, atoms prepared outside the trapping regions rapidly diffuse across the regions between them. Particles prepared in the trapping regions remain there. This regime occurs for $t^{*} \sim 1 /(K \epsilon)^{2}$ and corresponds approximately to the experimental results shown in Fig. 6.

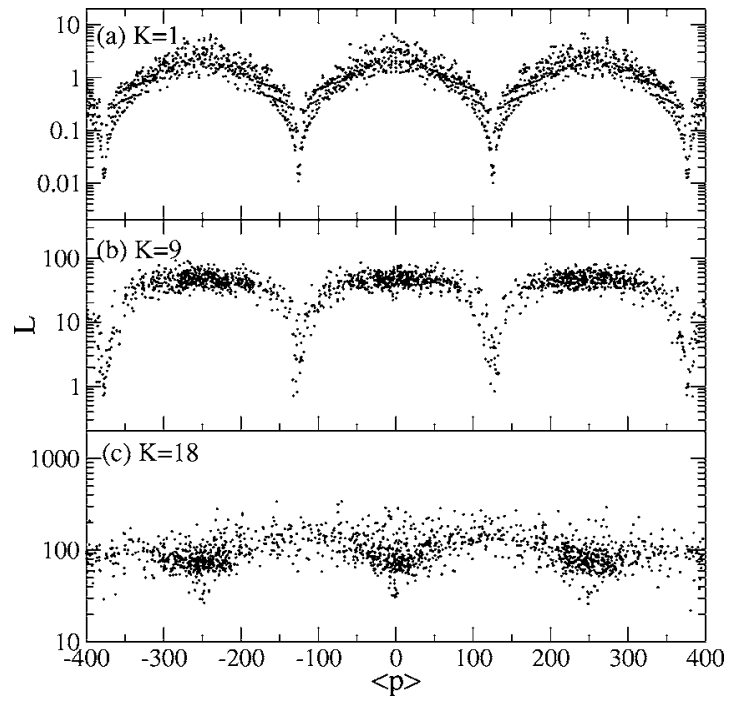

FIG. 7. Localization lengths of typical states for the double $\delta$-KR $(\epsilon=0.025, \hbar=0.5)$ corresponding to the three classical diffusion regimes investigated in the experiments in [11].

Finally, at the longest time scales, there is the $C_{G 1}$ correction investigated in [11], which is a long-ranged globalcorrelation family. $C_{G 1}$ results in an oscillation of the form $-\cos p_{0} \epsilon$ and becomes dominant at the longest timescales. The oscillation is of the same period as the one-kick correlation, but is of opposite sign. This means that at the longest time scales, the minima in energy absorption shown in Fig. 6(a) become maxima in energy absorption; and vice versa: the maxima become minima. Figure 6(c) shows experiments tending toward this regime. The inverted peaks of the Poisson family are still in evidence, but a $-\cos p_{0} \epsilon$ oscillation is clearly superposed. This is a somewhat counterintuitive result since it implies that atoms initially prepared in the momentum trapping regions are the ones which at long times, for $t^{*} \gg 1 /(K \epsilon)^{2}$, will absorb the most energy (there are no further reversals of this behavior at even longer times).

\section{Double $\delta$-KR: Floquet states}

The classical analysis thus reveals that there are three distinct classical diffusive regimes occurring at three time scales. We can expect that the corresponding quantum behavior will depend on which regime is dominant when dynamical localization arrests the quantum momentum diffusion. To investigate this, we now investigate how the form of the Floquet states varies in these different regimes.

In Fig. 7(a) we show the localization lengths of the Floquet states for a weak kicking strength. It can clearly be seen that the localization varies periodically as a function of momentum, staying within the range $1 \leqslant L \leqslant 10$ for the majority of points, with the exception of a series of sharp cusplike features at which the localization dramatically falls. The location of these cusps exactly corresponds to the "trapping momenta" $p \epsilon=(2 n+1) \pi$, predicted from classical arguments. The Floquet states centered in the trapping regions have widths of $L \simeq 0.01$, much narrower than states localized on stable islands, which are also visible in this figure as regular 


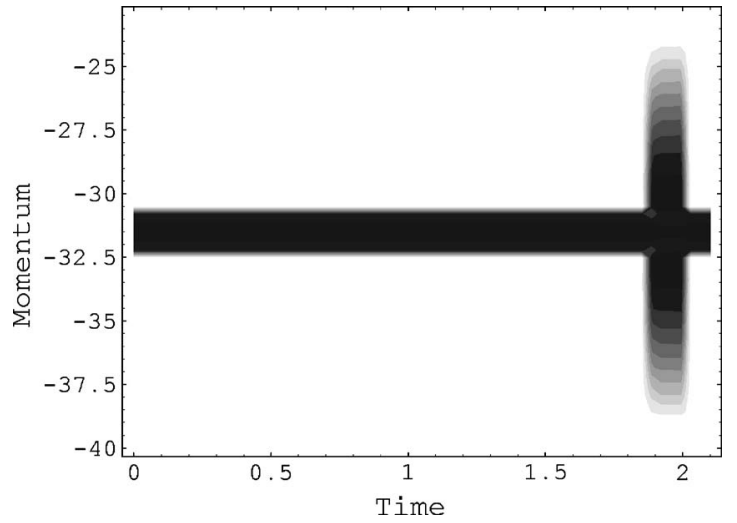

FIG. 8. Time evolution of a localized Floquet state in momentum space, $N(p, t)$, for physical parameters $K=2, \epsilon=0.1$, and $\hbar$ $=0.5$. Initially the Floquet state is sharply peaked at $p=-10 \pi$, in the center of a trapping region. The first kick at $t=1.9$ causes the state to spread across a much broader range of momentum, until the second kick at $T=2$ restores the localized state.

strings of points at $L \sim 1$. At the experimental values of $K$ $\simeq 3$ and $\epsilon=0.01$ a similar behavior is produced, with the broadest Floquet states having localization lengths of $L$ $\simeq 60$, while the narrowest have widths of $L \simeq 0.03$, over one thousand times narrower.

The Floquet states at the tips of the cusps have such low localization lengths that they are effectively pure plane-wave states (this can be further corroborated by evaluating the inverse participation ratio for these states, which indeed takes a value of almost unity). It is thus unsurprising that the presence of these states corresponds to the classical trapping effect, as a quantum system prepared in such a state will have a vanishingly small overlap with any other state and so will remain frozen (or trapped) in its initial state. It is important to note, however, that this quantum trapping effect depends critically on the order of the two kick periods-that is, whether the system is driven with a short-long kick sequence or the inverse long-short ordering.

This may appear surprising at first, since the Floquet states are periodic, with the same period $T_{\text {tot }}$ as the driving, and this period is not altered by interchanging the order of the kicks. Although it is frequently neglected, however, it is important to recall that the Floquet states do have an explicit time-dependence within each period, and this is able to produce substantially different behavior [18] when the phase of the driving field is altered. To illustrate this, we show in Fig. 8 the time evolution of one of the localized Floquet states, which experiences $\delta$ kicks at times $t=T_{1}=1.90$ and $t=T_{\text {tot }}$ $=2$. As can be seen, the state has only a trivial time evolution during the first time interval $\left(0 \leqslant t<T_{1}\right)$, since it is almost a plane wave and is thus approximately an eigenstate of the free Hamiltonian. The first kick at $T_{1}$ causes the wave packet to spread considerably in momentum space, before the second kick restores this broadened state to its original narrow form. Thus in this brief window of time between the two kicks, even the most localized Floquet states have a considerable spread in momentum. As a consequence, if the phase of the kicking field is shifted so that the system experiences the short-long kick sequence, none of the Floquet states are

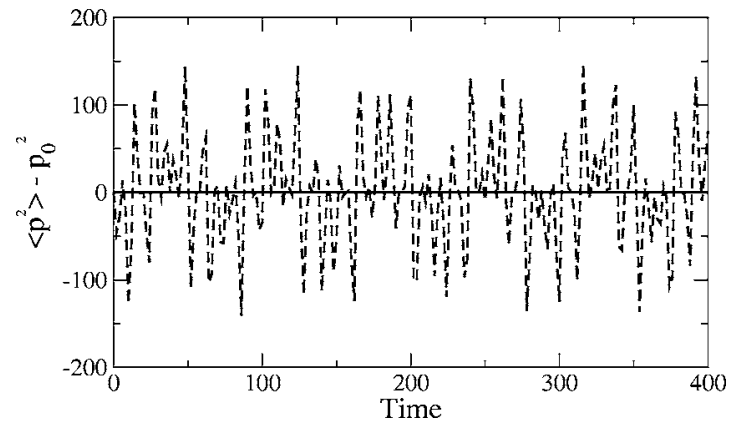

FIG. 9. Time evolution of the energy of the double $\delta$-KR, for the same physical parameters as in Fig. 7(a). The solid line shows the evolution of the system under the long-short kicking sequence, and shows few features. The dotted line shows the result of the shortlong sequence, and exhibits a complicated quasiperiodic behavior.

sharply localized in momentum at $t=0$. We show in Fig. 9 the time evolution of the system's kinetic energy when it is prepared in a momentum eigenstate in a trapping region. For the long-short kick sequence this state projects onto essentially a single Floquet state at $t=0$, and so its time evolution is trivial and its energy remains constant. For the case of the short-long kick sequence, however, the initial state projects onto a number of Floquet states [Eq. (3.2)], giving rise to a complicated quasiperiodic behavior arising from beating between the different quasienergies.

In Fig. 7(b), we see the effect of increasing the kick strength. In this regime we find there is an almost constant localization length for momenta in between the trapping regions, which are again signaled by sharp cusplike structures. This indicates that the Floquet states are confined between the classical broken phase barriers in the trapping regions. Early studies indicate that the level statistics of the corresponding quasienergies are not pure Poisson in this regime, as would be the case for the standard QKR.

In Fig. 7(c), we see an inversion of the broad momentum modulation in Fig. 7(a), similar to the reversal seen in the experiment. In this regime, the eigenstates localized in the trapping regions near $p \simeq(2 n+1) \pi / \epsilon$ are typically broader than those localized in between.

\section{CONCLUSIONS}

We have presented a study of the Floquet states of $\delta$-kicked particles pulsed with unequal periods, and used them to analyze experimental data on these systems. We conclude that the chaotic ratchet effect proposed in [7] and observed experimentally in [9] is associated with asymmetric Floquet states localized around $p=0$. We conclude also that the behavior of the localization lengths of the Floquet states for the double $\delta$-kicked rotor broadly accompany the transitions between the three distinct classical diffusion regimes investigated experimentally in [11].

\section{ACKNOWLEDGMENTS}

We would like to thank Mischa Stocklin for useful discussions, and the EPSRC for financial support. 
[1] G. Casati, B. V. Chirikov, F. M. Izraelev, and J. Ford, in Stochastic Behavior in Classical and Quantum Hamiltonian Systems, edited by G. Casati and J. Ford, Lecture Notes in Physics Vol. 93 (Springer, Berlin, 1979), p. 334; B. V. Chirikov, Phys. Rep. 52, 263 (1979).

[2] S. Fishman, D. R. Grempel, and R. E. Prange, Phys. Rev. Lett. 49, 509 (1982).

[3] F. L. Moore, J. C. Robinson, C. F. Bharucha, Bala Sundaram, and M. G. Raizen, Phys. Rev. Lett. 75, 4598 (1995).

[4] D. A. Steck, W. H. Oskay, and M. G. Raizen, Phys. Rev. Lett. 88, 120406 (2002).

[5] W. K. Hensinger, H. Häffner, A. Browaeys, N. R. Heckenberg, K. Helmerson, C. McKenzie, G. J. Milburn, W. D. Phillips, S. L. Rolston, H. Rubinsztein-Dunlop, and B. Upcroft, Nature (London) 412, 52 (2001).

[6] F. M. Izraelev, Phys. Rep. 196, 299 (1990).

[7] T. S. Monteiro, P. A. Dando, N. A. C. Hutchings, and M. R. Isherwood, Phys. Rev. Lett. 89, 194102 (2002).

[8] T. Jonckheere, M. R. Isherwood, and T. S. Monteiro, Phys. Rev. Lett. 91, 253003 (2003).
[9] P. H. Jones, M. Goonasekera, H. E. Saunders-Singer, and D. R. Meacher, quant-phys/0309149.

[10] P. H. Jones, M. Goonasekera, H. E. Saunders-Singer, T. S. Monteiro, and D. R. Meacher, physics/0504096.

[11] P. H. Jones, M. M. Stocklin, G. Hur, and T. S. Monteiro, Phys. Rev. Lett. 93, 223002 (2004);M. Stocklin, G. Hur, and T. S. Monteiro, physics/0408088.

[12] T. Dittrich, R. Ketzmerick, M.-F. Otto, and H. Schanz, Ann. Phys. (Leipzig) 9, 1 (2000); H. Schanz, M.-F. Otto, R. Ketzmerick, and T. Dittrich, Phys. Rev. Lett. 87, 070601 (2001).

[13] S. Flach, O. Yevtushenko, and Y. Zolotaryuk, Phys. Rev. Lett. 84, 2358 (2000).

[14] P. Reimann, Phys. Rep. 361, 57 (2002).

[15] D. Ł. Shepelyansky Phys. Rev. Lett. 56, 677 (1986).

[16] B. G. Klappauf, W. H. Oskay, D. A. Steck, and M. G. Raizen, Phys. Rev. Lett. 81, 1203 (1998).

[17] J. Ringot, P. Szriftgiser, J. C. Garreau, and D. Delande, Phys. Rev. Lett. 85, 2741 (2000).

[18] C. E. Creffield, Europhys. Lett. 66, 631 (2004). 